\title{
Computational Study of Human Calcitonin (hCT) Oligomer
}

\author{
Youngshang Pak, Jungho Shin, ${ }^{\dot{*}, a}$ and Soonmin Jang ${ }^{*}, \times$ \\ Department of Chemistry, Pusan National Lnmersity, Busan 609-735. Korea \\ ${ }^{\dagger}$ Department of Chemistry, Sejong Chiversity, Seoul 1 $13-747$, Korea. E-mail: sjangiásejong.ac.kn \\ Received January 29, 2008, Accepted October 21, 2009
}

\begin{abstract}
We have perfonmed long time REMD simulation on 15-19 residues of human calcitonin homone (DFNKF) which is known to fonm highly ordered anyloid fibril. The simulation started from randomly oriented multiple DFNKF strand. Using all-atom level sinulations with the generalized Bon solvation (GB) model (param99MOD3), we observed spontaneous formation of $\beta$-sheet for tetramer. Interestingly, the current simulation gives anti-parallel sheet as a major conformation, consistent with experiments. The major interaction stabilizing the anti-parallel sheet seems to be the inter-strand hydrogen bond
\end{abstract}

Key Words: Molecular dynamics simulation. Computational chemistry. Huntan calcitonin (hCT), Amyloid

\section{Introduction}

Over the last couple of decades there has been more than enough evidences showing that the formation of highly ordered protein (or peptide) aggregates are directly related to many pathologically distinct diseases. called amy loid disease. such as Alzheimer's disease, CJD (Creutzfeldt-Jacob disease), Parkinson's disease and Huntington's disease ${ }^{1-3}$ Several amyloidogenic proteins are isolated and characterized. ${ }^{4}$ Usually. these proteins aggregate to form insoluble fibril. known as amyloid fibril, and they have a characteristic crossed beta sheet structure perpendicular to the fibril axis. suggesting there could be common mechanistic pathway's toward the diseases. Therefore. a detailed understanding of insoluble protein aggregates is of fundamental importance for therapeutic purposes. So far. however, little is known of its detailed structure and formation mechanism. This is mainly due to experimental difficulties in conventional structural determination methods such as NMR and $\mathrm{X}$-ray because the aggregate is not only insoluble but also it hardly forms single crystal. It is noteworthy that recent advances in various alternative experimental techniques. such as $\mathrm{X}$-ray with micro single crystal, by which the GNNQQNY structure of yeast prion protein Sup 35 has been determined. ${ }^{5}$ and solid state NMR. ${ }^{6}$ shed light on the structural information of amy loid fibrils. Thus. some of the common features of fibril have begun to emerge. At present. however. numerous structural and dy namic characteristics including the detailed structure of general amyloid fibril, its formation mechanism, and the minimum size of critical nucleus seed that may acts as a template for fibril growth largely remain unknown.

Unlike experiments. carefully designed computational studies may give direct and detailed information on protein aggregates and the aggregation process. thereby complementing experimental studies. This has prompted many researchers in computational biochemistry over the past several years to perform numerous calculations on properties of amy loid fibrils.

${ }^{2}$ Current address, Korea Institute of Science and Technology, Seoul, 136-791, Korea including structure characterization. stabilities. and its formation pathways ${ }^{8.9}$ One of the central areas of focus has been the detailed structure of each fibril and the main driving force toward the self assembly of amyloidogenic proteins into the final stable beta sheet connected structure. It seems the zipper is a conmon motif for sheet-sheet recognition.

The human calcitonin (hCT) hornone is one of the amyloidogenic proteins with 32 -amino acids and its amyloid formation is related to medullary carcinoma of the thyroid. ${ }^{10}$ Experimentally' peptide with residues 15-19 (DFNKF) also forms stable antyloid fibril. ${ }^{11,12}$ However. the detailed structure with atomic level is not known to date despite the simplicity of its constituting peptide. Recently, a series of molecular dynamics simulation in Nussinov group tried to address the stability and important interactions for this amyloid fibril. ${ }^{13-16}$ Using molecular dynamics simulations at ligh temperature with various possible arrangements. they found that the stable structure of aggregates is a parallel $\beta$-sheet even though the experimental data implicates the existence of a large portion of an anti-parallel structure. ${ }^{12}$ They noticed the electrostatic interaction between $\mathrm{C}$ terminus and Lys and between $N$ terminus and Asp plays important role along with hydrogen bond network between Asn side chains in their parallel $\beta$-sheet arrangement. Also. they noticed the Asn-Asn interaction plays major role in DFNKF stability and formation

Over the past several years, the focus of amy loidosis study has shifted from fibrilar form to oligomers since oligomers are not only the intermediates for fibril formation but in most cases also have higher cell toxicity than the fibril itself. ${ }^{19.20}$ In terms of the conputational requirements. the oligomerization of peptide is very slow process even with today"s high perfornance computer. Thus. direct simulation of oligomerization is rarely feasible unless some efficient simulation strategy is employed. In this paper. we performed computer simulation of the aggregation of hCT oligomers (tetramer) through efficient sampling scheme. the replica exchange molecular dynamics (REMD) simulation. ${ }^{13.18}$ In REMD. molecular dy namics simulation is performed with several independent trajectories at different temperatures simultaneously. Then the trajectory exchange 
Table 1. The REMD temperature distribution and the conesponding replica exchange acceptance. Acceptance at $300 \mathrm{~K}$ indicates acceptance between $288 \mathrm{~K}$ and $300 \mathrm{~K}$

\begin{tabular}{|c|c|c|c|c|c|c|c|c|c|c|c|c|}
\hline Temp. (K) & 288 & 300 & 315 & 332 & 352 & 374 & 398 & 423 & 450 & 478 & 508 & 540 \\
\hline Acceptance $(\%)$ & & 39.9 & 33.7 & 28.8 & 21.1 & 21.6 & 24.5 & 28.3 & 300 & 31.4 & 31.5 & 34.7 \\
\hline
\end{tabular}

between neighboring temperature are allowed at every given time interval and this can greatly enhance the overall sampling efficiency since the high temperature trajectories tend to explore configuration space better than the low temperature trajectories. Unlike the previous computational studies by Tsai et al. ${ }^{1+4}$ who performed a short REMD simulation of hCT trimer starting from putative stable parallel $\beta$-sheet conformation. we performed self-assembly of the hCT tetramer starting from randomly oriented monomers. Considering the time scale of most amyloid fibril formation. computational simulation of formation of the ordered hCT aggregates is a daunting task but this approach can give indirect information on the formation mechanism and stability of hCT oligomers as well as possible aggregated oligomer structures.

Even though the stability of oligomeric hCT conformers are addressed by extensive stability test that start from various possible arrangements. ${ }^{13.15}$ there could be other possible arrangements especially as the number of hCT strands increases. The reason we performed REMD simulation starting from constituting monomers is two fold. First. what are the possible stable conformers when hCT aggregates into oligomer and what is their relative stability? Second, are the resulting conformers consistent with previous simulations and experiments? Tetramer may be good candidate for this purpose since oligomers of this size can exhibit more variations in monomer arrangement to form $\beta$-sheet connected oligomer than simple dimer or trimer.

\section{Methods}

The initial conformation of each monomer is extended and the $\mathrm{N}$ terminus and $\mathrm{C}$ termimus of each monomer is patched with acetyl group (Ace) and N-methyl group (Nme) respectively. Multiple strands of extended DFNKF monomers are placed inside the simulation box with a distance of about $20 \AA$ between each strand. Then. the system is heated to $600 \mathrm{~K}$ for $500 \mathrm{ps}$ followed by local energy minimization. Please note that this will generate completely random initial configuration. The simulation box consists of an imaginary sphere with a radius of $25 \AA$ from the center of mass. If the atoms fall out of this imaginary boundary. the atoms will experience an attractive harmonic force toward the center of mass of the system with a force constant of $1.0 \mathrm{kcal} \cdot \mathrm{mol}^{-1} \cdot \mathrm{A}^{-2}$. This will effectively confine the whole sy'stem and prevent from the molecules flying apart from each other, thus mimicking a cellular enviromment. Even with REMD, self assembly of small peptides with all atom level is computationally challenging. especially under the presence of explicit solvation. In an attempt to reduce the overall computational requirement while considering the reasonable solvation effect. we used the generalized Born (GB) solvation model with a modified version of all atom amber99 force field (param99MOD3) that we have developed previously. ${ }^{23}$ Most all atom force fields tend to exhibit somewhat unbalanced $\alpha / \beta$ propensity with over-stabilized salt bridge effects within the GB solvation framework. The param99MOD3 modified force field is a result of extensive enpirical tuning of the parang9. MOD2 force field by Onufriev et al ${ }^{2+}$ such that the global minimunt of free energy landscape obtained from REMD sinulation corresponds to the native state for several training sets. i.e. $\alpha \beta \beta$ nini-proteins (IFSD. IPSV. and BBA5) which are structurally subtle and conputationally challenging. It has been shown that the resulting force field can describe free energy profile of several structurally distinct peptides reasonably.

The temperature range for the replica exchange was set to $288 \mathrm{~K}-540 \mathrm{~K}$ with 12 replicas as listed in Table 1 . The replica exchange interval was $0.16 \mathrm{ps}$. This provides an average replica exchange acceptance of about $29 \%$. We implemented the REMD scheme into the Tinker ${ }^{26}$ mol ecular dynamics simulation package using version 4.2 and used stochastic dynamics with friction coefficient $1.0 \mathrm{ps}$ throughout this study. Note that the friction constant we used is smaller than the actual water friction constant $(79 \mathrm{ps}$ at $300 \mathrm{~K}$ ) to enllance the sampling speed and this will not change the overall sinulation results. ${ }^{2,28}$ The simulation time step was $2.0 \mathrm{fs}$ with fixed bond distances between hydrogen atoms and heavy atons using RATTLE. ${ }^{29}$ Considering the time scale of peptide aggregation. the self assembly sinulation requires extensive computing even with very efficient sampling methods such as the REMD we used here. The total REMD simulation time for each replica was 136 ns. resulting in total $136 \times 12=1632 \mathrm{~ns}$. The cut distance of both the non-bonded interaction and the GB solvation was set to $24 \AA$. The trajectory was saved at every $400 \mathrm{ps}$ and the last $32 \mathrm{~ns}$ of REMD simulation results were used for further analysis and production purposes.

\section{Results and Discussion}

First. we checked the overall performance of the REMD sinulation. Frequently, the clange of the replica index as a function of simulation time is used as a criteria for this purpose. ${ }^{21.23]}$ Following the history of all trajectories. the replica exchange index of one of the selected trajectories for tetramer simulation was obtained as a function of the REMD simulation time and it is shown in Figure 1. The trajectory visits all replicas from the lowest temperature (index 1 ) to the highest temperature (index 12), indicating the trajectory is stochastic enough with reasonable trajectory mixing within the overall REMD simulation time. In addition to the good trajectory mixing. reasonable convergence is required to observe the overall ensemble of trajectories at given temperature. We have performed backbone RMSD (root mean square deviation) based hierarchical cluster analy sis ${ }^{32}$ at every $8 \mathrm{~ns}$ of REMD simulation time (20000 conformers) during the whole simulation time ( $136 \mathrm{~ns})$ and calcu- 


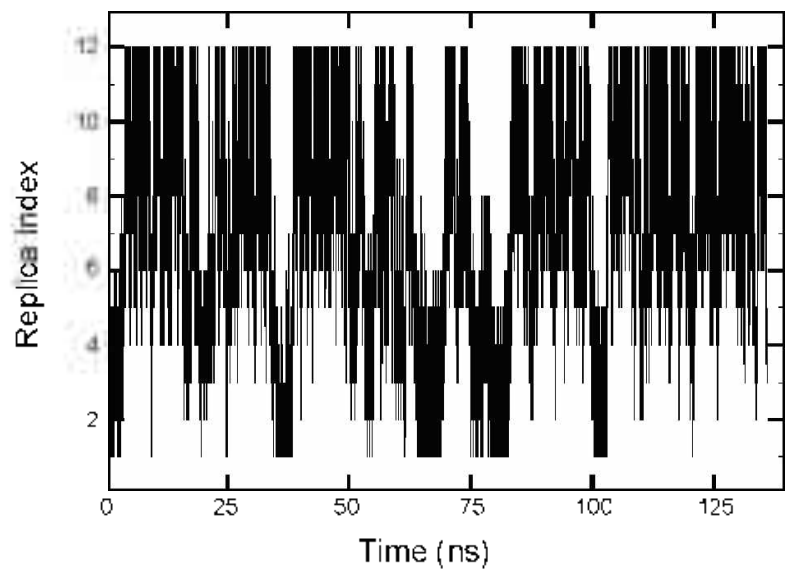

Figure 1. 'The replica index ol one of the trajectories for the teramer simulation as a lunetion of IRI:MD time. Other trajectories show similar behavior. The trajectory visits all replieas many times during the sinulation, indieating the sistem is stodkatsic and reasonable trajector mixing has been achieved.

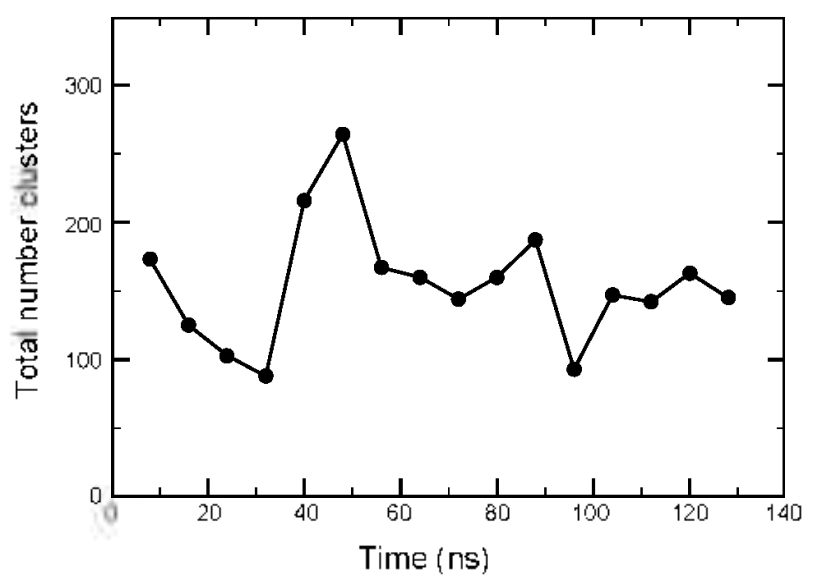

Figure 2. The total number of eluster at $300 \mathrm{~K}$. 'The number of eluster

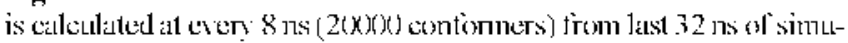
lation time. $A$ s the RMSl) erileria increases, the number of cluster decreases in general. After slow inerease of total eluster number. it

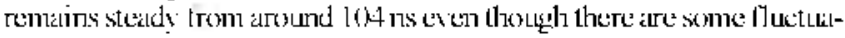
tions. "lhe figure indicales (ha RI:MI) simulation has renched elose to the equilibrium.

lated the total number of cluster to monitor the REMD convergence $^{3.3}$ at $300 \mathrm{~K}$. In hicrarchical clustering analysis. for cach conformer. the RMSD with the rest of conformer is calculated and the number of conformers having RMSD value less than the given critcria (close neighbor) is counted. After that. the conformer with the highest number of this close neighbor (central conformer) is removed from the initial collection of structure. along with those close neighbors. and classified as single clusier. The same procedure is repeated with remaining conformers until no conformer is left. The number of total cluster at cicry 8 ns with RMSD criteria of $5.0 \mathrm{~A}$ is plotted for last 32 ns of REMD simulation in Figure 2. The number of total cluster is fluctuating as simulation proceeds and it scems it remains stcady after 104 ns. suggesting the REMD has reached to quasi-cquilibrium. For the final structural analysis. we dis-

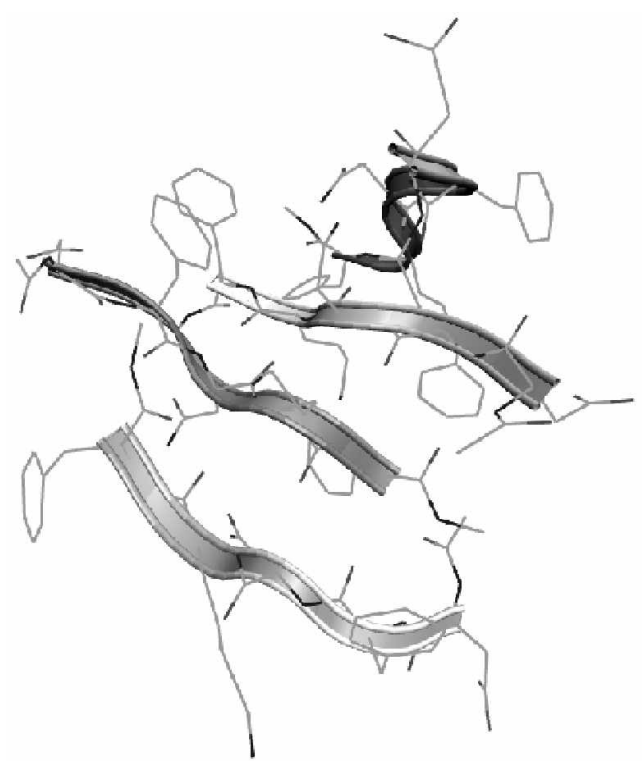

Figure 3. The representative structure of the mest populated cluster. The hydrogen honds are represented by dotted lines. The side chains are also shown in stick fonn for clárity.

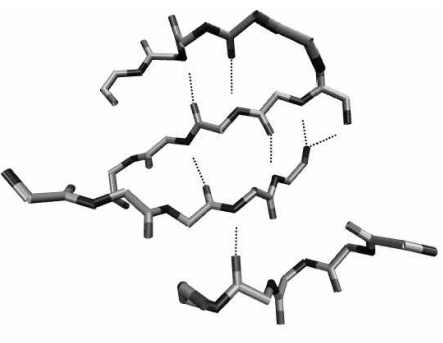

$17.4 \%$

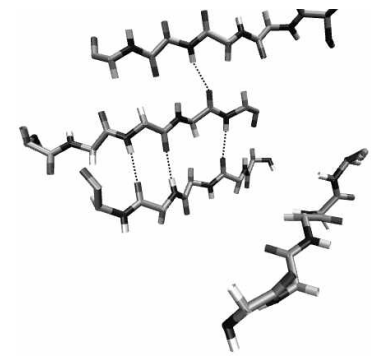

$11.6 \%$
Figure 4. Representative struclures of two elusters (clusker $2 \sim$ eluster 3 ) next to the highest populated eluster (cluster I). The values indicate 1he population of each oluster. Note the highest populated tuster cluster lo represents $40.4 \%$ of the total population.

carded initial 104 ns trajectory and performed hicrarchical clustering analy sis with trajectories from last $32 \mathrm{~ns}$. One of the main results of present study comes from this clustering analysis at $300 \mathrm{~K}$. When we used RMSD of $5,0 \mathrm{~A}$ as clustering criteria. the relative populations of first seven clusters are $40.4 \% .17 .4 \%$ $11.6 \%$ and $3.7 \%$. The top four highest populated clusters took $66.1 \%$ of total frames analy $/ \mathrm{ed}$. The most highly populated clusier ( $40.4 \%$ of all total conformers) has a proto-fibril like single layer $\beta$-sheet with anti-parallel arrangement. This finding is different from previous reports on stability simulations. in which parallel $\beta$-shect is the main stable structure. ${ }^{15}$ The representative structure of mosi populated clusier for tetramer simulation is shown in Figure 3. The side chains are also shown as stick form. The representative structure is taken as the central conformer of the cluster during clustering analy sis. The three strands forms stable $\beta$-sheet while the forth strand s sheet formation is rather loose. The structure shows a rig/ag pattem by one residue in a sense that the end of each strand is not 


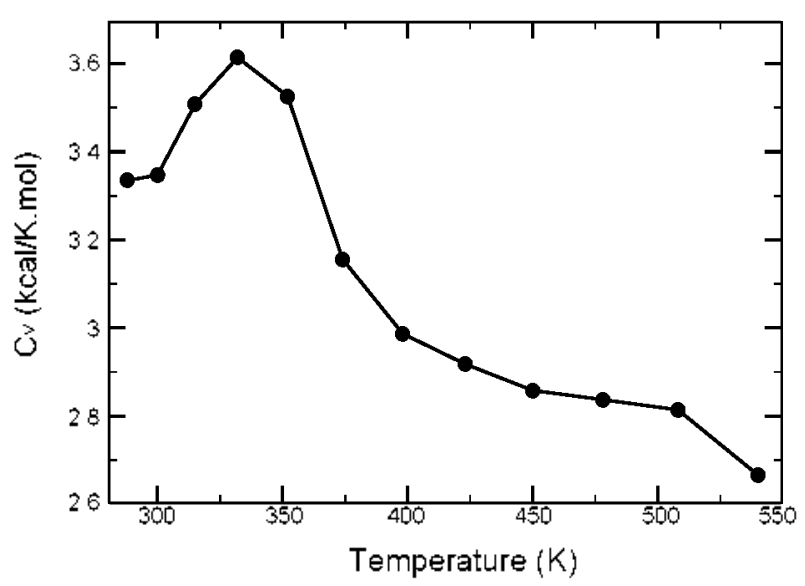

Figure 5. The constant volume heat capacities at all REMD replica temperature. The phase transition temperature is located at the heat capacity peak at around $330 \mathrm{~K}$.

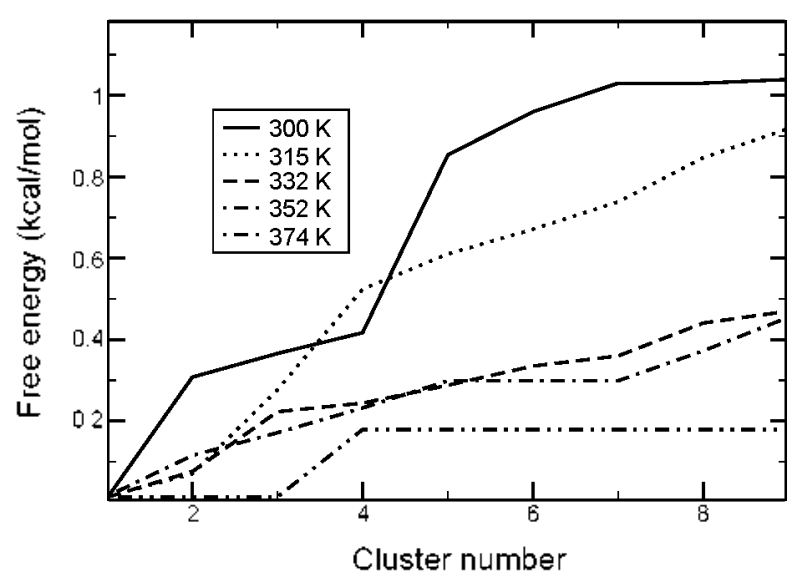

Figure 6. The free energy landscape as a function of discrete coordinate of cluster number at several temperatures. The free mergy is relative energy.

linearly aligned. This is possibly due to inter-strand Phe-Phe $\pi-\pi$ interaction with anti-parallel direction since the Phe residues are at second place from $\mathrm{N}$-terminial and first place from C-terminal. As for this inter-strand $\pi-\pi$ stacking the geometry shows it is not optimally placed. resulting in a weak $\pi-\pi$ interaction. It seems the interstrand hydrogen bonds are a major factor in stnictural stability. Note that in the parallel $\beta$-sheet conformation of Tsai et al. ${ }^{15} \pi-\pi$ stacking also appears and its $\pi-\pi$ stacking tendency is somew hat higher than current conformation. The interstrand backbone hydrogen bonds are between Ly's and Ly's. Phe-Phe. and Asn-Phe. In addition to the backbone hydrogen bonds. fluctuating hydrogen bonds between Asn side chains also appeared. The structure of the next two clusters. each laving population of $17.4 \%$ and $11.6 \%$ respectively. are basically composed of three strand $\beta$-sheet like + monomer, possibly intermediates for the formation of well aligned anti-parallel tetramer strand (Figure 4). The inter-stand hydrogen bonds are indicated with dotted lines. Here, we considered the hydrogen bond is "formed" if the bond distance is within $3.5 \AA$ and the bond angle is larger than $150^{\circ}$. We did not observe any parallel strands in this tetramer simulation. The current simulation clearly shows anti-parallel $\beta$-sheet is the stable and dominant conformer for hCT oligomers. consistent with the experiment. ${ }^{12}$

We calculated the energy fluctuation. and thereby the constant volume heat capacities at all twelve REMD simulation temperatures. The heat capacity as a function of temperature in Figure 5 indicates that it exlibit phase transition at around $330 \mathrm{~K}$, which corresponds to collapse of anti-parallel sheet. Thus. it is expected that this anti-parallel beta strand can survive up to around $330 \mathrm{~K}$. The same tendency can be observed from the free energy landscape as a function of the discrete coordinate of cluster number in Figure 6 . In this coordinate, cluster number 1 has the highest number of population. At $300 \mathrm{~K}$, the free energy difference between the highest populated (anti-parallel $\beta$-sheet like structure) and the random structure (cluster number 9) is more than $1.0 \mathrm{kcal} / \mathrm{mol}$. But from around $332 \mathrm{~K}$. the free energy difference is less than $0.5 \mathrm{kcal} / \mathrm{mol}$, suggesting that the antiparallel $\beta$-sheet can disintegrates due to thermal fluctuation.

The trajectories obtained from REMD sinulation represent ensemble of conformation at given temperature, lacking any direct dynamic information. Therefore. it is not the purpose of present work to probe the detailed assenbly pathway of anyloid like hCT(15-19). Instead. the main focus of current study is finding the stable oligomer structure(s) connected with $\beta$-sheet that might be related to further aggregation into proto-fibril with no. or minimal simulation bias/constraint imposed on the system. Possibly the largest Due to this limitation. the current simulation might not capture the water hydrogen bond network in the hCT fibril, which might play an inportant role in fibril stabilization. "since the simulation was performed under the GB implicit solvation environment. Therefore, in the future it needs to be studied further the stability and detailed water hydrogen bond network of the structure obtained from current simulation in the presence of explicit water using extensive simulations.

In terns of free energy. the aggregation of the hCT nonomer into oligomer will increase free energy initially since the entropy decrease will dominate the enthalpy increase. But as the size of the oligomer increases. at some point, the enthalpy will dominate entropy and free energy will decrease as the oligomer size increases. In general. an oligomer of this size will serve as a nucleus seed for further growth into proto-fibril. Neither the size of this oligomer nor the structure is known at this time. As for the hCT. the size of this critical nucleus seed could be small. ${ }^{15}$ It would be interesting to calculate and compare the free energy of oligomerization for hCT by changing the oligomer size sy'stematically including the two oligomers we presented here even though each calculation requires considerable computational resources.

\section{Conclusions}

The detailed structure of the 15-19 segment of the hCT hormone protein, which forms amyloid fibril, is unknown with atomistic details. Recent computational studies show that the parallel beta-sheet strand might be the stable form. This con- 
tracts to the experimental data which indicate that the antiparallel beta-sheet is the major stable structure depending on the solution $\mathrm{pH}^{1 / 2}$ In an effort to understand this structural difference we performed the REMD simulation of acetyl and methylamine patched 15-19 segment of hCT hormone starting from fully extended structure with random orientation with all atom level using the GB implicit solvation model (param99MOD3).

The simulation results indicate that the anti-parallel amy loid proto-fibril like $\beta$-sheet is the major conformer. stabilized mainly by interstrand hydrogen bonds. In general, the formation of amyloid fibril is very sensitive to the environments such as concentration. temperature, and $\mathrm{pH}$. The solvation model we adapted is implicit solvent rather than explicit water molecules. which require a great amount of computational resources. This might be the main limitation for the simulation presented in this study. Clearly, the anti-parallel $\beta$-sheet structure from present simulation needs further study with more elaborated model including explicit solvent simulations. Furthermore the mechanistic details such as the main driving force and pathway of this oligomer formation possibly using hydrogen bond network analysis approach such as study by Tsai et al. ${ }^{14}$ may contribute to understanding the somewhat subtle structural and dy namic features of hCT (15-19).

Acknowledgments. SJ appreciates financial support from Sejong University.

\section{References}

1. Kelly, J. W. Cument Opinton in Strtictwal Biolog, 1998, 8, 101.

2. Hardy, J.: Selkoe, D. I. Science 2002, 297, 353.

3. Chiti, F.: Dobson, C. M. Anmal Review of Biochemistry 2006, 75,333 .

4. Westemark, P. Amyloidosis and imwoid Protems: Brief History and Defnaitions; WILEY-VHC: 2005: Vol. 1.

5. Nelson, R.: Sawaya, M. R.; Balbinie, M.: Madsen, A. O.: Riekel, C.: Grothe, R: Eisenberg, D. Nature 2005, 435,773

6. Tycko, R. Biochentistry 2003, 42,3151.

7. Zheng, T. Ma, B. Y : Nussinov, R. Phisical Biologv $2006,3, \mathrm{Pl}$.

8. Caflisch, A. Current Opinion in Chemical Biologv 2006, 10, 437

9. Lee, S.; Kim, Y. Bulletin of the Korean Chemical Society 2004, 25,838 .
10. Zaidi, M.: Inzerillo, A. M: Moonga, B. S.: Bevis, P. I. R.: Huang. C. L. H. Bone 2002, 30,655.

11. Reches, M.; Porat, Y.; Gazit, E. Jom of Biological Chemistn 2002, 277, 35475.

12. Naito, A.: Kamihira, M.; Inoue, R.; Saito, H. Hagnetic Resonance in Chemistiv 2004, $\$ 2,247$.

13. Tsai, H. H. G.; Tsai, C. J.; Ma, B.; Gunasekaran, K; Zanuy, D.; Nussinov, R. Biophusical Joumal 2004, 86,412A.

14. Tsai, H. H; Reches, M.; Tsai, C. J.; Gunasekaran, K.; Gazit, E; Nulssinov, R. Proceedings of the National Academ of Sciences of the Cinited States of America 2005, 102,8174.

15. Tsai, H. H.; Zanuy, D.; Haspel, N.; Gunasekaran, K.; Ma, B. Y.; Tsai, C. T.; Nussinov, R. Biophysical Jom omal 2004, 87, 146.

16. Haspel, N.: Zanuy, D.: Ma, B. Y.: Wolfson, H.: Nussinov, R. Jomnal of Holecular Biolog, 2005, 3*5, 1213.

17. Sugita, Y: Okamoto, Y. Chemt. Phys. Lett. 1999, 31 t, 141.

18. Swendsen, R. H.; Wang, J. S. Phys Rev. Lett. 1986, 57, 2607.

19. Walsh, D. M.: Klyubin, I.: Fadeeva, J. V.; Cullen, W. K.; Anwy1, R.; Wolfe, M. S.; Rowan, M. J Selkoe, D. J. Nature 2002, 116 , 535.

20. Klein, W. L.; Kraft, G. A.; Finch, C. E. Trends in Kenrosciences $2001,24,219$.

21. Gsponer, I.: Haberthur, U.; Caflisch, A. Proceedings of the Nationat Acalem of Sciences of the Lnited States of America 2003 , 100,5154 .

22. Beglov, D.: Roux, B. Jommal of Chemical Phusics 1994, 100 , 9050 .

23. Jang. S.: Kim, E.: Pak, Y. Proteins-Stnwctme Function and Bioinfomatics 2006, 62,663

24. Onufriev, A.: Bashford D.: Case, D. A. Proteins-Strtcture Function and Bioinfomatics 2004, 55, 383.

25. Jang, S.: Kim, E.: Pak, Y. Proteins-Structure Function and Bioinformatics 2007, 66, 53 .

26. Ponder, J. W. TINKER 4.2 : software tools for molecular design: Washington University, 2004

27. Zagrovic, B Sorin, E. J Pande, V. Joumal of Molecular Biology $2001,313,151$

28. Homak, V; Okur, A; Rizzo, R. C.; Simmerling, C. Proceedings of the Vational Acadenv of Sciences of the Lnited States of Anterica 2006, 103,915

29. Palmer, B. J. Joumat of Contutational Physics 1993, 104, 470.

30. Rao, F Catlisch, A. Joumal of Chentical Physics 2003, 119, 4035.

31. Periole, X.; Mark, A. E. Joumal of Chemical Physics 2007, 126.

32. Daura, X.; Gademann, K.; Jaum, B.; Seebach, D.; van Gunsteren, W. F.; Mark, A. E. Angewandte Chemie-Inemational Edition $1999,38,236$.

33. Affentranger, R; Tavemelli, I.; Di Iorio, E. E. Joumal of Chentical Theon and Computation 2006, 2, 217 . 